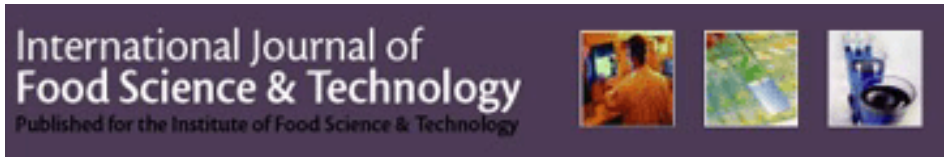

\title{
Quality characteristics of pasta enriched with buckwheat flour
}

\begin{tabular}{|r|l|}
\hline Journal: & International Journal of Food Science and Technology \\
\hline Manuscript ID: & Draft \\
\hline Manuscript Type: & Original Manuscript \\
\hline Keywords: & Pasta, Buckwheat Flour, Texture, Image Analysis, Cooking \\
\hline \multicolumn{2}{|l}{} \\
\hline
\end{tabular}

\section{SCHOLARONE ${ }^{m}$ \\ Manuscripts}


1 Title: Quality characteristics of pasta enriched with buckwheat flour

2 Authors: Alessandra Marti, Lorenzo Fongaro, Micaela Rossi, Mara Lucisano, M. Ambrogina 3 Pagani* $^{*}$

4 DiSTAM, Università degli Studi di Milano, via G. Celoria 2, 20133 Milan, Italy

$5 \quad$ * corresponding author: $\quad$ ambrogina.pagani@unimi.it 


\begin{abstract}
1 Abstract:
2 The addition of pseudocereal flours to semolina is becoming more and more popular to improve the

3 nutritional quality of the resultant pasta. The aim of this study was the property evaluation of pasta

4 made on industrial scale from a mixture of buckwheat flour and durum wheat semolina. The

5 characterization of samples belonging to different pasta producers took into account the evaluation

6 of chemical properties, pasting behavior, water uptake at $25{ }^{\circ} \mathrm{C}$ and $90{ }^{\circ} \mathrm{C}$, mechanical properties of

7 the dry product, cooking behavior (cooking losses, weight increase, instrumental firmness and

8 adhesiveness) and surface characteristics monitored by image analysis. The product characterization

9 highlighted high heterogeneity of the mechanical properties of the uncooked and cooked products

10 (breaking stress: $17.57 \div 43.81 \mathrm{~N} / \mathrm{mm}^{2}$; adhesiveness: $5.49 \div 7.38 \mathrm{~J}$ ), solid loss into cooking water $11(4.25 \div 4.99 \%)$, and water absorption $(80.65 \div 92.95 \%)$. The great variability may be due to the 12 different raw-materials and processing conditions adopted by each pasta producer.

13 Key words: pasta, buckwheat flour, texture, image analysis, cooking
\end{abstract}


1 1. Introduction

2 Semolina from durum wheat is recognized to be the most suitable raw material for pasta production 3 due to its unique colour, flavor, and cooking quality (De Noni and Pagani, 2010). The reasons for

4 the present success and diffusion of pasta also depends on the possibility of using other flours (such 5 as oat and barley), pseudocereals (such as buckwheat) and legumes (such as lentils), alone or mixed 6 with semolina, which are interesting from the point of view of fibre and/or protein sources, in order 7 to satisfy the demand for healthy food.

8 Buckwheat is characterized by an excellent nutrient profile thanks to its high protein quality, the 9 presence of high amounts of fibre, vitamins, and minerals (Aubrecht and Biacs, 2001), and 10 digestible starch, which makes this pseudocereal recommended for diabetics (Edwardson, 1996). It 11 is also a source of bioactive compounds (flavonoids, sterols, etc.) with health-promoting effects 12 (Aubrecht and Biacs, 2001). Thus, the use of buckwheat flour in the formulation of high quality, 13 healthy products such as pasta (Rayas-Duarte et al., 1996; Alamprese et al., 2007), bread (Fessas et 14 al., 2008; Lin et al., 2009), and biscuits (Baljeet, et al., 2010) is increasingly attracting the attention 15 of food scientists.

16 With regards to Italy, the most famous dish made with buckwheat flour is Pizzoccheri, obtained 17 from a mixture of buckwheat flour, durum wheat semolina, and coarse semolina (Pagani et al., 18 2007). In the artisan process, an aliquot of buckwheat flour (generally from 20 to 25\%) is added to 19 fine and coarse semolina, and the dough (about 35\% moisture) is formed into Pizzoccheri by 20 sheeting (tagliatella-shaped; width less than $1 \mathrm{~cm}$ ) and consumed within 1-2 days. On the industrial 21 scale, this type of pasta can be prepared by using different technologies: the dough can be formed 22 by sheeting or by extrusion; in addition, the extrusion and sheeting steps can be combined. As it can 23 be assimilated to a dried pasta, its quality can be evaluated considering different features related 24 both to the characteristics of the dry product, such as colour, fracturability, surface appearance and 59 evenness, and to cooking behavior in terms of cooking loss, consistency, and adhesiveness of the cooked product (D'Egidio et al., 1990; Lucisano et al., 2008). The addition of buckwheat flour in 
1 relevant percentages (30-50\%) to wheat semolina or flour to produce dry pasta is responsible for the

2 increased fragility of the pasta structure, a higher loss of organic matter into the cooking water and a

3 different texture, compared with semolina pasta (Rayas-Duarte et al., 1996; Manthey et al., 2004).

4 No information is available on the overall characteristics of Pizzoccheri, a product traditionally

5 made by small companies that follow their own production rules and trust their own experience.

6 Moreover, these producers are interested in obtaining the Protected Geographical Indication (PGI)

7 denomination for Pizzoccheri. This denomination expresses a strong bond between the product and

8 the region (EEC Council Regulation No. 2081/92). Nevertheless this dependence does not require

9 that all phases of the transformation process be performed in a particular geographical area. A PGI 10 product nonetheless complies with strict production regulations established for the process, and 11 compliance with these regulations is assured by inspection.

12 The objective of the present work is to give a complete overview of Pizzoccheri features made by 13 the three most important industrial Pizzoccheri producers currently present in Italy. The 14 characteristics of Pizzoccheri products were evaluated both in their raw state and after cooking 15 using conventional and innovative approaches in order to understand the physical arrangement and 16 the macromolecular interactions in each sample and describe the heterogeneity of these commercial 17 products. In fact, this wide characterization could provide useful information for obtaining the PGI 18 denomination for Pizzoccheri.

19 2. Material and method

$20 \quad 2.1$ Pizzoccheri samples

21 Three different Pizzoccheri brands (coded A, B, and C) were bought from the Italian market. For 22 each brand, three batches were purchased from three different stores, and analysed. The samples 23 coded B and C were produced in the Valtellina valley, a small area in the north of Italy. The sample 24 coded A was produced in Lombardy, outside of the Valtellina area.

Both sample A and C were formed into short-strands pasta, with an average length of $67.1 \mathrm{~mm}$ and $50.5 \mathrm{~mm}$, respectively (Table 1). Brand B was formed into long-shape pasta (like skein pasta) and 
1 for this reason it was cut into pieces of $6 \mathrm{~cm}$ before analysis (Figure 1). The width of the samples 2 ranged from $7.40 \mathrm{~mm}$ for sample $\mathrm{B}$ to $7.81 \mathrm{~mm}$ for sample $\mathrm{C}$; and the thickness varied from 1.42 $3 \mathrm{~mm}$ (sample B) to $1.52 \mathrm{~mm}$ (sample C).

42.2 Chemical composition

5 Before chemical analyses, Pizzoccheri were ground with a laboratory mill (IKA Universalmuhle 6 M20, Janke and Kunkel GmbH \& CoKG, IKA Laborteknic, Staufen Germany) to particles size less 7 than $500 \mu \mathrm{m}$. The moisture and ash content of the pasta samples were determined according to 8 official standard methods AACC 44-15 and AACC 08-12 (2001). The total nitrogen content was 9 evaluated following the official standard method AOAC 920.87 (1999); a factor of 5.7 was used to 10 convert the nitrogen content to protein content $(\% \mathrm{db})$. All these evaluations were made at least in 11 triplicate. Total starch was determined enzymatically using the "Total Starch Assay Kit" (AACC 12 76-13, 2001; Megazyme International Ireland Ltd., Bray Business Park, Bray, Co. Wicklow, 13 Ireland). The damaged starch content refers to the amount of starch that, being quickly susceptible 14 to $\alpha$-amylase hydrolysis, had been mechanically damaged during milling. In the this work, the enzymatic approach was used to characterize the samples in an attempt to assess the influence of the technological process on starch organization. Susceptibility to $\alpha$-amylase was determined using the “Starch Damage Assay Kit” (AACC 76-31, 2001; Megazyme International Ireland Ltd., Bray 18 Business Park, Bray, Co. Wicklow, Ireland). The results are the average of a minimum of four 19 replicates.

\subsection{Pasting properties}

21 The pasting profiles of ground pasta were carried out according to Mariotti et al. (2005), using a 22 MicroVisco-Amylo-Graph (MVAG) (Brabender OHG, Duisburg, Germany). An aliquot of $12 \mathrm{~g}$ 23 was dispersed in $100 \mathrm{~mL}$ of distilled water, scaling both flour and water weight on $14 \%$ flour 24 moisture basis. The pasting properties were evaluated under constant conditions (speed: $250 \mathrm{rpm}$; sensitivity: $300 \mathrm{~cm} \mathrm{~g}$ ) using the following time-temperature profile: heating from $30{ }^{\circ} \mathrm{C}$ up to 95 
1 cooling from $50{ }^{\circ} \mathrm{C}$ to $30^{\circ} \mathrm{C}$. The heating and cooling phases were carried out with a temperature 2 gradient of $3{ }^{\circ} \mathrm{C} / \mathrm{min}$. The analysis was repeated at least twice and the parameters of pasting 3 properties were determined using the software provided with the instrument (Viscograph version $4 \quad 2.3 .7)$.

\subsection{Hydration test}

6 An aliquot of $10 \mathrm{~g}$ of samples was placed in a beaker containing $200 \mathrm{ml}$ of water (pasta:water ratio $7=1: 20)$ at $25^{\circ} \mathrm{C}$ and $90{ }^{\circ} \mathrm{C}$. These temperatures were maintained constant placing the beakers in a 8 thermostatic bath. After $5,10,15,30,60$, and 180 minutes at $25^{\circ} \mathrm{C}$, and $2,5,7,10,12,15,30$, and 945 minutes at $90{ }^{\circ} \mathrm{C}$, Pizzoccheri were removed from the water, drained for one minute, carefully 10 blotted with tissue paper to remove superficial water, and weighed. The analysis was repeated for 11 each batch at least twice. The results were expressed as $\left(\mathrm{W}_{1}-\mathrm{W}_{0}\right) * 100 / \mathrm{W}_{0}$, where $\mathrm{W}_{1}$ is the weight 12 of the hydrated sample and $\mathrm{W}_{0}$ is the weight of the dry sample.

\subsection{Solid content of cooking water and water absorption}

14 Cooking loss was evaluated by determining the amount of solids lost into the cooking water 15 (D'Egidio et al., 1990). An aliquot of $50 \mathrm{~g}$ of pasta was cooked in $500 \mathrm{ml}$ of boiling natural spring 16 water (pasta:water ratio $=1: 10$ ) with no salt added. Pasta samples were cooked until the optimum 17 cooking time (OCT) suggested by each companies and reported in the label: 12 minutes for sample 18 A; 10 minutes for sample B; 15 minutes for sample C. After cooking, the volume of water was 19 brought to the initial volume. Dry matter was determined on $25 \mathrm{ml}$ of cooking water, dried to 20 constant weight at $105^{\circ} \mathrm{C}$. The residue was weighed, reported as percentage of the dry material, and 21 expressed as grams of matter loss/100 $\mathrm{g}$ of pasta.

22 The increase of pasta weight due to cooking was evaluated by weighing pasta before and after 23 cooking. The results were expressed as $\left(\mathrm{W}_{1}-\mathrm{W}_{0}\right) * 100 / \mathrm{W}_{0}$, where $\mathrm{W}_{1}$ is the weight of cooked pasta 24 and $\mathrm{W}_{0}$ is the weight of the uncooked sample. 
1 The texture properties of Pizzoccheri were evaluated before and after cooking using a Texture 2 Analyzer TA-HD (Stable Micro Systems, Surry, UK). A calibrated load cell of $500 \mathrm{~N}$ was used for 3 the collection of data (500 points per second).

4 The fracture properties of the uncooked samples were evaluated by a three-point bending test with 5 the appropriate holder (HDP/3PB - Three Point Bend) having a $61 \mathrm{~mm}$ span length. A portion of 6 sample with a length of about $5 \mathrm{~cm}$ was put on the holder and was deformed up to the broken point 7 with a blade (speed: $10 \mathrm{~mm} / \mathrm{s}$ ). Measurements were replicated on 30 strands for each brand. Strain 8 and stress were calculated from the force-distance curve. In particular, strain at the breaking point 9 (ع) was calculated according to Bruns and Bourne (1975): $\varepsilon=6 * \mathrm{D}^{*} \mathrm{~d} / \mathrm{L}^{2}$, where $\mathrm{D}$ is the depth of 10 sample $(\mathrm{mm})$; $\mathrm{d}$ is the distance at the break point $(\mathrm{mm})$, and $\mathrm{L}$ is the distance between the support $11(61 \mathrm{~mm})$. The stress $\left(\sigma ; \mathrm{N} / \mathrm{mm}^{2}\right)$ corresponding to the stress required to break the sample was 12 calculated by the following expression: $\sigma=3 \mathrm{PL} / 2 \mathrm{bD}^{2}$, where $\mathrm{P}$ is the load at the break point $(\mathrm{N}), \mathrm{L}$ 13 is the distance between the support $(61 \mathrm{~mm}), \mathrm{b}$ is the sample width $(\mathrm{mm})$, and $\mathrm{d}$ is the depth of 14 sample (mm).

15 The compression test performed on the cooked samples was made according to the method 16 "Adhesive testing for cooked pasta". For this purpose 6 strands of Pizzoccheri were cooked at the 17 OCT, cooled for 20 minutes and placed in a support block HDP/PFS - Pasta Firmness stickiness 18 (Stable Microsystems, Surry, UK). This support blocks the Pizzoccheri strands while leaving the 19 possibility of access to the square piston to compress the product. Sample strands were compressed 20 with a speed of $1 \mathrm{~mm} / \mathrm{s}$ up to a load of $50 \mathrm{~N}$ and maintained compressed at the same load for 3 21 seconds, before removal of the load. The test was repeated 7 times for each sample. From the 22 compression curve the following parameters were obtained and considered as indicator of product 23 stickiness: Young Modulus $\left(\mathrm{N} / \mathrm{mm}^{2}\right)$, Adhesiveness $(\mathrm{J})$ corresponding to the negative area of force24 time curve $(\mathrm{N} * \mathrm{~s})$. 
1 The images of 6 Pizzoccheri for each batch of each commercial brand were taken before and after 2 cooking at their OCT, using a flatbed scanner (Epson Perfection 3170 Photo, Seiko Epson Corp., 3 Japan), at $600 \mathrm{dpi}$ (dots per inch) of resolution and a colour depth of 24 bits in standard conditions.

4 The images were saved as TIFF format and then processed using a dedicated software (Image Pro$5 \quad$ Plus 4.5.1.29, Media Cybernetics Inc, UK).

6 The assessment of surface texture of uncooked and cooked products was performed on a surface of $7 \quad 501 \mathrm{pxl} * 101 \mathrm{pxl}$ extracted from the images of the Pizzoccheri and was used for creating the data set 8 of 108 images for samples, 54 for uncooked and 54 for cooked products. After conversion in 8-bit 9 grayscale, the surface texture of each image was evaluated and expressed in terms of heterogeneity 10 (HTG). This parameter is defined as the fraction of pixels whose intensity value deviates more than $1110 \%$ compared to the average intensity of the entire image. A heterogeneity value equal to 0 12 corresponds to a homogeneous surface (smooth surface); on the other hand, a value equal to 1 13 corresponds to a heterogeneous surface (rough surface).

142.8 Statistical analysis

15 The data were processed by STATGRAPHIC ${ }^{\circledR}$ Plus for Windows v. 5.1. (StatPoint Inc. Virginia, 16 U.S.A.). A one-way analysis of variance (ANOVA) was performed using the Least Significant 17 Differences (LSD) test to compare the sample means; differences were considered significant at $18 \mathrm{p}<0.05$

19 3. Results and discussion

\subsection{Pizzoccheri characterization}

21 All samples were prepared by using coarse semolina, buckwheat flour and durum wheat semolina 22 as the main ingredients. Only brands $\mathrm{A}$ and $\mathrm{C}$ stated the amount of buckwheat flour added to the 23 semolina (25\% for both samples). Due to the commercial origin of the products, the composition of 24 raw materials was unknown. 
1 The chemical composition of Pizzoccheri is shown in Table 1. The protein content ranged from 211.7 to $13.5 \%$ d.b., in every case higher than the minimum fixed by Italian law for conventional 3 pasta from durum wheat semolina. Therefore, the integration of semolina with buckwheat flour 4 assured a good protein amount, as the most currently grown cultivars yield seeds with 11-15\% 5 protein (Mazza and Oomah, 2005). Sample B showed the lowest starch content $(67.8 \%$ d.b.), 6 considerably lower than the amount declared in label, and a higher ash content probably related to 7 the use of less refined flours. No significant difference in either the protein or starch content was 8 detected between sample A and C (Table 1).

9 Italian law establishes the maximum moisture and ash values only for dried pasta from durum 10 wheat semolina (Italian law $\mathrm{n}^{\circ} 580,4$ July 1967 modified by the presidential decree $\mathrm{n}^{\circ} 187,9$ 11 February 2001). Even if no indication has been proposed for Pizzoccheri pasta, all samples had 12 moisture contents below $10.55 \%$ (Table 1), similar to the semolina products. The ash content of the 13 samples ranged from 1.09 (sample A) to 1.64\% d.b. (sample B). The significant differences between 14 sample B and the other two brands can be related to the quantity and/or the quality of the raw 15

materials, as processing seems not to affect this parameter (Manthey and Hall, 2007). Regardless of the nature of the flours, the mineral content in all the samples is higher than in semolina pasta (max

$170.90 \%$ d.b., according to the Italian law). At the same time, the ash content in Pizzoccheri was lower 18 compared to whole wheat pasta (1.35\% according to Olivera and Salvadori, 2006). Even if the ash 19 content in buckwheat seeds (1.37-1.67\% d.b.) is lower than in wheat, semolina-buckwheat mixture 20 contained more ash than semolina alone, as reported by Manthey and Hall (2007). The addition of 21 buckwheat flour at $25 \%$ replaced minerals removed by milling durum wheat into semolina 22 (Manthey and Hall, 2007).

233.3 Starch properties

The damaged starch content represents the amount of starch granules suitable to being quickly hydrolyzed by $\alpha$-amylase as a consequence of physical modifications to the native structural 26 organization. In the case of dry pasta, the damaged starch is a marker of the physical changes 
1 induced by thermal treatments, such as extrusion and drying (Marti et al., 2010; Mariotti et al., 2 2011).

3 According to previous works (Mariotti et al., 2006; Lacavalla et al., 2010), the amount of damaged

4 starch due to wheat and buckwheat milling is usually lower than 3-4\% (buckwheat 1.5-2.2\%).

5 Therefore, the higher values observed for Pizzoccheri demonstrate that starch granules undergo 6 relevant physical stresses during pasta-making, especially during the forming and drying phase. The 7 damaged starch content varied from $10.9 \%$ of sample $\mathrm{C}$ to $11.3 \%$ of sample A (Table 2). The 8 process conditions used to prepare sample $\mathrm{C}$ seem to promote a more compact structure of the 9 product, as argued by the lower $\alpha$-amylase susceptibility.

10 The starch modifications detected by the enzymatic hydrolysis with $\alpha$-amylase could also be 11 responsible for changes in the pasting properties of starch granules. Changes in viscosity of 12 Pizzoccheri during heating and cooling are shown in Table 2. This approach is conventionally 13 adopted for the evaluation of pasting properties of starch and flours. In this work, investigating the 14 pasting properties of dry pasta could give information on molecular changes promoted by pasta15 making process (Marti et al., 2010; Mariotti et al., 2010). All the samples showed a typical 16 viscoamylographic curve, with an increase in viscosity during the heating step, a decrease in 17 viscosity during the prolonged heating of the suspension (evaluated by the breakdown index), and 18 an increase in viscosity during the cooling period (evaluated by the setback index) (Table 2). 19 Sample B showed the lowest pasting temperature (Table 2), probably as a consequence of the 20 lowest amount of total starch and the highest protein content in this sample (Table 1). Moreover, 21 this behaviour could be attributed to changes to the molecule arrangement that occurred during 22 processing and resulting in a product in which starch granules showed a higher ability to absorb 23 water and swell when heated in excess of water. On the other hand, starch present in samples A and 24 C appeared to be more rigid to water access and more stable during the heating step (low 25 breakdown index). During the holding period at $95{ }^{\circ} \mathrm{C}$, the product slurries were subjected to high temperatures and mechanical shear stress which further disrupted starch granules, resulting in 
1 amylose leaching out. This period is commonly associated with a breakdown in viscosity, which 2 was higher in sample B than in the other two Pizzoccheri brands (Table 2). During cooling, the 3 viscosity increased as a result of the formation of a gel structure. This phase is commonly described 4 as the setback region and is related to the re-association between starch molecules, especially 5 amylose. During cooling, sample B showed the lowest final viscosity and the lowest setback value, 6 indicating a lower rate of starch retrogradation, probably related to the use of less refined flours 7 (lower starch and to the higher protein content of this sample) (Santos et al., 2008) (Table 2). Even 8 if samples $\mathrm{A}$ and $\mathrm{C}$ had a similar behavior during the heating phase, sample $\mathrm{C}$ reached the highest 9 peak viscosity (Table 2).

\subsection{Hydration test}

11 The ability of pasta products to absorb water, i.e. the weight gain during cooking, is affected by 12 both raw material composition and processing conditions. Moreover, it is considered to be one of 13 the most important characteristics for a producer of Pizzoccheri pasta (personal communication). 14 The kinetics of water uptake of Pizzoccheri samples at $25^{\circ} \mathrm{C}$ and $90{ }^{\circ} \mathrm{C}$ are shown in Figure 1a and $151 \mathrm{~b}$, respectively, for times longer than the optimal cooking time (Table 4) with the aim of better 16 investigating the porosity of the samples.

17 Water absorption at room temperature $\left(25^{\circ} \mathrm{C}\right)$ can be related both to the presence of hydrophilic 18 macromolecules, such as fibre, and to the samples' structural features determined by the technology 19 adopted during Pizzoccheri production. In fact, starch gelatinization and protein coagulation, which 20 accompany the cooking at $90{ }^{\circ} \mathrm{C}$, could certainly mask the macrostructure characteristics of the 21 products (Maache-Rezzoug and Allaf, 2005). Sample B had the highest water uptake capacity at 22 any sampling time (Figure 2a). Even after 5 minutes of soaking in water, the amount of water 23 absorbed by sample B was higher than that absorbed by samples A and C. A similar trend, with a 24 significant difference, was observed after 10 minutes of soaking. The increase of the soaking time that sample B had significantly different absorption ability in comparison with the other two 
1 commercial brands $(\mathrm{p}<0.05)$. A less compact structure and a high porosity could be responsible for

2 the faster penetration of water in sample B. On the other hand, the lower amount of water absorbed

3 by sample $\mathrm{C}$ suggests a greater compactness of the sample with, potentially, a lower extent of

4 leaching phenomena during cooking.

5 The absorption kinetics at $90{ }^{\circ} \mathrm{C}$ showed that samples $\mathrm{A}$ and $\mathrm{B}$ absorbed a higher amount of water

6 than sample C, at any soaking time (Figure 2b). The high water absorption capacity of sample B

7 confirmed its higher swelling ability exhibited by the micro-viscoamylographic test, in comparison

8 with samples A and C (Table 3). No significant difference was observed between sample A and B

9 up to 12 minutes of soaking. In addition, the higher the soaking time, the higher the differences 10 among them $(\mathrm{p}<0.05)$. This behavior could be related to the macromolecular changes induced by 11 heat that occurred close to the optimal cooking time of each Pizzoccheri brand (Table 3). The 12 technological history of the samples seemed to be responsible for the peculiar structure of the 13 product that resulted in a lower absorption capacity for the sample C. Moreover, the hydration 14 capacity could account for the choice of the cooking time recommended by each Pizzoccheri 15 producers: the lower the water absorption, the longer the cooking time.

163.3 Solid content of cooking water and water absorption

17 Cooking losses are considered a useful indicator of overall spaghetti cooking performance 18 (D'Egidio et al., 1990). The solid losses of Pizzoccheri were within the range (4.3\% - 5.0\%) (Table 193 ) and they are comparable to those of conventional pasta from semolina and whole semolina pasta 20 (4.2\% and 3.6\%, respectively; data not shown). The addition of non-gluten forming flours diluted 21 the gluten network and interrupted and weakened the overall structure of pasta, allowing leaching of 22 soluble solids into the cooking water (Pagani, 1986). Moreover, the high amount of fibre contained 23 in Pizzoccheri (2.7\%d.b.) accounted for the weakening of the gluten network and for the slight 24 increase of cooking losses. Buckwheat bran contains 12\% soluble fibre (Steadman et al., 2001) and part of it leaches out of the products during cooking (Manthey et al., 2004). The amount of buckwheat (25\%) added to durum wheat did not dramatically increase the amount of solid losses, 
1 despite the dilution of the gluten network created by buckwheat proteins. Similar results were 2 obtained in a previous work, in which the authors concluded that since pasta from buckwheat flour 3 has cooking losses equal or lower than pasta made from semolina alone, it presented excellent 4 cooking characteristics (Chillo et al., 2008).

5 Sample A was able to absorb the highest amount of water (93\%) and, at the same time, to release 6 the highest quantity of material into the boiling water (5\%). This behavior was probably due to a 7 looser pasta structure at its optimal cooking time (12 minutes). The result confirmed the differences 8 in structure compactness detected during the hydration test at $90^{\circ} \mathrm{C}$ : the water uptake for sample A, 9 B and C was respectively $93 \%, 81 \%$ and $86 \%$ after soaking for the optimal cooking time of each 10 brand (12, 10 and 15 minutes for sample A, B, and C, respectively) (Figure 1a).

\subsection{Texture properties}

12 The texture properties of pasta before and after cooking are summarized in Table 3. The three commercial brands presented significant differences in breaking strength before cooking $(\mathrm{p}<0.05)$.

14 In particular, sample $\mathrm{C}$ exhibited higher strain and stress values at rupture, compared to samples $\mathrm{A}$ 1

and $\mathrm{B}$. The compactness of sample $\mathrm{C}$ reflected its behavior in cold and hot water: the lower the

16 fragility, the lower the absorption values (Figure 1). It is well known that the breaking strength of 17 pasta is highly dependent on the die type (Lucisano et al., 2008) and the extrusion conditions 18 (Pagani et al., 1989). The low breaking stress and strain of sample A in its dry state is indicative of 19 a loose internal structure, accounting for high water adsorption and cooking losses (Table 3).

20 Even after cooking, sample $\mathrm{C}$ showed a higher firmness than sample A. On the other hand, no 21 significant differences were detected between samples A and C for adhesiveness (Table 3). The 22 higher values of Young modulus detected in samples B and C can be related to the starch and 23 protein arrangement in the Pizzoccheri products, accounting for the low amount of water absorbed 24 during cooking (91 and 89\%, respectively) and for the lower amount of starch released in cooking water (4.3 and 4.5\%, respectively) compared to sample A. Regarding adhesiveness, sample B exhibited the lowest values, and consequently the best cooking behaviour. 
13.5 Surface texture characteristics by Image Analysis

2 Surface texture is often used to describe the surface characteristics of a material (Zheng et al.,

3 2006). It is a parameter immediately perceived by sight, and it anticipates a specific tactile

4 perception (Chen, 2007). In this study, the heterogeneity (HTG) parameter was used to describe the

5 surface texture of the samples before and after cooking, in order to gain information on the degree

6 of roughness and, consequently, on the product structure (Figure 3).

7 The HTG before and after cooking is shown in Figure 4. In all samples, a decrease in HTG was 8 detected after cooking, highlighting that cooking increased surface homogeneity $(\mathrm{p}<0.05)$. The 9 surface roughness of the dry products is related to the conditions applied during the pasta shaping 10 step, while starch and its physiochemical properties certainly play an important role in structural 11 changes during cooking. In particular, before cooking, sample A had a more heterogeneous surface 12 than samples $B$ and $C(p<0.05)$, showing a rough and uneven surface, features that can easily be 13 perceived even by simple observation and tactile assessment of the product (Figure 3). It is worth 14 noting that sample A also presented a lower resistance to fracture indicating a looser internal 15 structure that is probably one of the reasons for the irregular surface. On the contrary, sample B 16 showed a smoother and less wrinkled surface. The different surface properties could be related to 17 the processing conditions (forming roller, type of die, etc.) (Lucisano et al., 2008).

18 The surface properties are one of the products characteristics that may help to explain pasta cooking 19 behaviour: the rougher the surface of the uncooked products, the higher the solid content of cooking 20 water (Table 4). In fact, samples with high HTG value (roughness surface) expose a greater area to 21 water action during cooking and, consequently, a high amount of material can be released on the 22 pasta surface and, consequently, into the cooking water.

23 Regarding the change in HTG values after cooking, samples A and C were characterized by a 24 greater decrease in the HTG index compared to sample B (66\% and 20\%, respectively), suggesting 59 6025 that cooking slightly affected the roughness of product B. 
1 The 3D representation of the grey level surface distribution (Figure 3) can help to forecast the 2 sensation perceived by the consumer: a rough and uneven surface in the uncooked samples, and a 3 smoother surface after cooking.

4 4. Conclusions

5 The results obtained by different and complementary approaches provided an overall 6 characterization of pasta enriched with buckwheat flours, commercialized by the three most 7 important Pizzoccheri producers currently present in Italy. The three products showed highly 8 different properties which may be due not only to the raw material characteristics (mainly semolina 9 quality) but also to the processing conditions adopted by each producers. Presumably, brand B 10 focuses on the choice of good quality raw materials (semolina with high protein content), providing 11 a product with low solid loss in cooking water and low adhesiveness. Sample A, on the contrary, is 12 characterized by the worst performance during cooking (high cooking losses and adhesiveness) that 13 can be related to a lower protein content and, consequently, a discontinuous structure. The 14 processing conditions adopted for both samples are effective in creating a porous network that is 15 able to absorb a higher amount of water than sample C. On the other hand, sample $\mathrm{C}$ is obtained by using a technology suitable for creating a more compact structure. These observations enable us to

17 hypothesize different technological processes for each sample. In the extrusion process commonly 18 used for semolina pasta production, the dough is submitted to significant shear stresses, which can 19 weaken the protein structure (Pagani et al., 1989), but, at the same time, to pressure (9-10 MPa) 20 responsible for giving the product a high compactness, allowing it to withstand cooking (Petitot et 21 al., 2009). On the other hand, the sheeting process by rolls allows the protein to align in a 22 continuous network whose goodness strongly depends on protein quantity and quality. Further 23 studies are underway to better understand the effect of both raw material characteristics and 24 processing conditions (sheeting and/or extrusion) on the physical properties and cooking quality of 25 buckwheat enriched pasta. 
1 Alamprese, C., Casiraghi, E., \& Pagani, M.A. (2007). Development of gluten-free fresh egg pasta

2 analogues containing buckwheat. European Food Research and Technology, 225, 205-213.

3 American Association of Cereal Chemists (AACC) (2001). Approved Methods of the AACC, St 4 Paul, MN, USA.

5 Association of Official Analytical Chemists (AOAC) (1999). Official Methods of Analysis, XVI 6 Ed, Gaithesburg, MD, USA.

7 Aubrecht, E., \& Biacs, P.A. (2001). Characterization of buckwheat grain proteins and its products. $8 \quad$ Acta Alimentaria, 28, 261-268.

9 Baljeet, S.Y., Ritika, B.Y., \& Roshan, L.Y. (2010). Studies on functional properties and 10 incorporation of buckwheat flour for biscuit making. International Food Research Journal, 17, $11 \quad 1067-1076$.

12 Bruns, A.J., \& Bourne, M. (1975). Effect of sample dimension on the snapping force of crisp food. 13 Journal of Texture Studies, 6, 445-458.

14 Chen, J. (2007). Surface texture of foods: perception and characterization. Critical Reviews in Food 15 Science and Nutrition, 47, 583-598.

16 Chillo, S., Laverse, J., Falcone, P.M., Protopapa, A., \& Del Nobile, M.A. (2008). Influence of the 17 addition of buckwheat flour and durum wheat bran on spaghetti quality. Journal of Cereal Science, $18 \quad 47,144-152$.

19 D’Egidio, M.G., Mariani, B.M., Nardi, S., Novaro, P., \& Cubadda, R. (1990). Chemical and 20 technological variables and their relationship: a predictive equation for pasta cooking quality. 21 Cereal Chemistry, 67, 275-281. 
1 De Noni I., \& Pagani M.A. (2010). Cooking properties and heat damage of dried pasta as 2 influenced by raw material characteristics and processing conditions. Critical Reviews in Food 3 Science and Nutrition, 50, 465-472.

4 EEC (European Economic Community) (1992). Council Regulaion No. 2081/92, 14 July 1992. 5 Official Journal of European Communities L208, 24 July 1992.

6 Edwardson, S. (1996). Buckwheat: pseudocereal and nutraceutical. In: Janick, J., (Ed). Proceedings 7 of the Third National Symposium New Crops: New Opportunities, New Technologies (pp 195-207). 8 ASHS Press, Alexandria, VA, USA.

9 Fessas, D., Signorelli, M., Pagani, M.A., Mariotti, M., Iametti, S., \& Schiraldi, A. (2008). 10 Guidelines for buckwheat enriched bread. Thermal analysis approach. Journal of Thermal Analysis 11 and Calorimetry, 91, 9-16.

12 Lacavalla, M, Lucisano, M., Mariotti, M., \& Ng., P.K.W. (2010). Influence of extrusion processing 13 conditions on the properties of buckwheat products. Cereal Food World, 55, A55.

14 Lin, L.Y., Liu, H.M., Yu, Y.W., Lin, S.D., \& Mau, J.L. (2009). Quality and antioxidant property of 15 buckwheat enhanced wheat bread. Food Chemistry, 112, 987-991.

16 Lucisano, M., Pagani, M.A, Mariotti, M., \& Locatelli, D.P. (2008). Influence of die material on 17 pasta characteristics. Food Research International, 41, 646-652.

18 Maache-Rezzoug, Z., \& Allaf, K. (2005). Study of the effect of hydrothermal process conditions on 19 pasta quality. Journal of Cereal Science, 41, 267-275.

20 Manthey, F.A., Yalla, S.R., Dick, T.J., \& Badaruddin, M. (2004). Extrusion Properties and Cooking 21 Quality of Spaghetti Containing Buckwheat Bran Flour. Cereal Chemistry, 81, 232-236. 
1 Manthey, F. A., \& Hall, C. A. (2007). Effect of processing and cooking on the content of minerals 2 and protein in pasta containing buckwheat bran flour. Journal of the Science of Food and 3 Agriculture, 87, 2026-2033.

4 Mariotti, M., Zardi, M., Lucisano, M., \& Pagani, M.A. (2005). Influence of the heating rate on the 5 pasting properties of various flours. Starch/Stärke, 57, 564-572.

6 Mariotti, M., Alamprese, C., Pagani, M.A., \& Lucisano, M. (2006). Effect of puffing on 7 ultrastructure and physical characteristics of cereal grains and flours. Journal of Cereal Science, 43, $8 \quad 47-56$.

9 Mariotti, M., Iametti, S., Cappa, C., Rasmussen, P., \& Lucisano, M. (2011). Characterization of 10 gluten-free pasta through conventional and innovative methods: evaluation of the uncooked 11 products. Journal of Cereal Science, accepted.

12 Marti, A., Seetharaman, K., \& Pagani, M. A. (2010). Rice-based pasta: a comparison between 13 conventional pasta-making and extrusion-cooking. Journal of Cereal Science, 52, 404-409.

14 Mazza, G., \& Oomah, B. D. (2005). Buckwheat as a food and feed. In: Abdel-Aal, E. and Wood, P. 15 (Eds), Specialty grains for food and feed (pp. 375-393). St Paul MN: American Association of 16 Cereal Chemists, Inc.

17 Olivera, D. F., \& Salvadori, V. O. (2006). Textural characterisation of lasagna made from organic 18 whole wheat. International Journal of Food Science \& Technology, 41, 63-69.

19 Pagani, M.A. (1986). Pasta products from non-conventional raw materials. In: Mercier, C., 20 Cantarelli, C., (Eds). Pasta and extrude products. Elsevier applied science, London.

21 Pagani, M.A., Resmini, P., \& Dalbon, G. (1989). Influence of the extrusion process on 22 characteristics and structure of pasta. Food Microstructure, 8, 173-182. 
1 Pagani, M.A., Lucisano, M., \& Mariotti, M. (2007). Traditional Italian products from wheat and 2 other starchy flours. In: Hui, Y.H. (Ed). Handbook of food products manufacturing (pp. 328-388).

3 John Wiley \& Sons, Inc., Hoboken, NJ, USA.

4 Petitot, M. Abecassis, J., \& Micard, V. (2009). Structuring of pasta components during processing: 5 impact on starch and protein digestibility and allergenicity. Trends in Food Science \& Technology, $6 \quad 20,521-532$.

7 Rayas-Duarte, P., Mock, C.M., \& Satterlee, L.D. (1996). Quality of spaghetti containing 8 buckwheat, amaranthus, and lupin flours. Cereal Chemistry, 73, 381-387.

9 Santos, E., Rosell, C. M., \& Collar, C. (2008). Gelatinization and retrogradation kinetics of high10 fiber wheat flour blends: a calorimetric approach. Cereal Chemistry, 85, 455-463.

11 Steadman, K.J., Burgoon, M.S., Lewis, B.A., Edwardson, S.E., \& Obendorf, R.L. (2001). 12 Buckwheat seed milling fractions: Description, macronutrient composition and dietary fibre. 13 Journal of Cereal Science, 33, 271-278.

14 Zheng, C., Sun, D.W., \& Zheng, L. (2006). Recent applications of image texture for evaluation of 15 food qualities-a review. Trends in Food Science and Technology, 17, 113-128. 
1 Table 1. Geometrical and chemical characterization of Pizzoccheri samples.

2 Table 2. Damaged starch and pasting properties of Pizzoccheri samples.

3 Table 3. Cooking and textural properties of Pizzoccheri samples.

4 Figure 1. Pizzoccheri shape.

5 Figure 2. Absorption kinetics at $25^{\circ} \mathrm{C}$ (a) and $90{ }^{\circ} \mathrm{C}$ (b) of Pizzoccheri samples.

6 Figure 3. Sample images: colour images of Pizzoccheri before (a) and after (d) cooking; 2D and 3D 7 representation of grey level surface distribution before $(b ; c)$ and after cooking $(e ; f)$.

8 Figure 4. Heterogeneity of Pizzoccheri before and after cooking. 
1 Table 1. Geometrical and chemical characterization of Pizzoccheri samples.

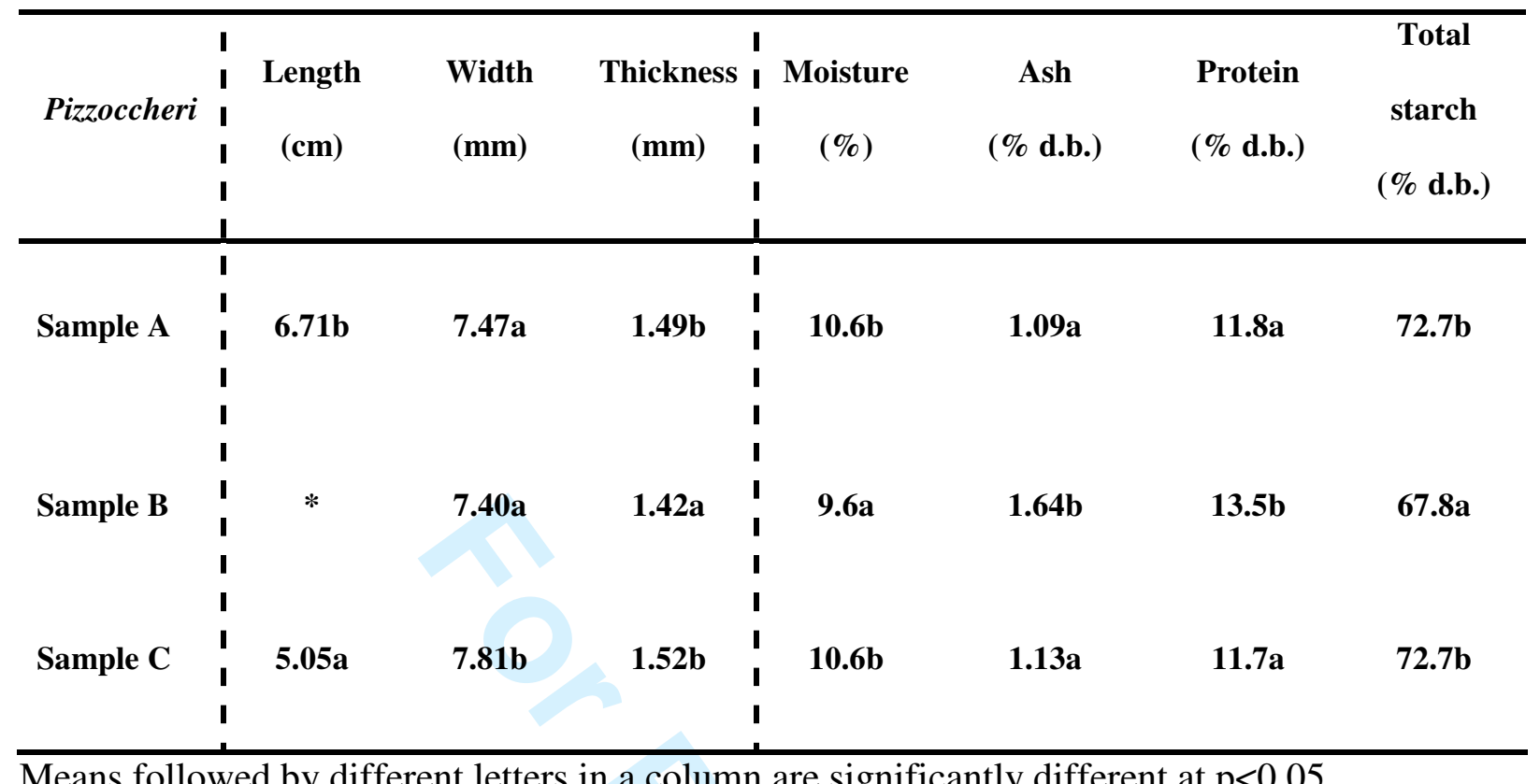

2 Means followed by different letters in a column are significantly different at $\mathrm{p}<0.05$.

3 *nest shaped Pizzoccheri 
1 Table 2. Damaged starch and pasting properties of Pizzoccheri samples.

\begin{tabular}{|c|c|c|c|c|c|c|}
\hline Pizzoccheri & $\begin{array}{c}\text { Damaged } \\
\text { starch } \\
\text { (\% d.b.) }\end{array}$ & $\begin{array}{c}\text { Pasting } \\
\text { Temperature } \\
\left({ }^{\circ} \mathbf{C}\right)\end{array}$ & $\begin{array}{c}\text { Peak } \\
\text { Viscosity } \\
\text { (BU) }\end{array}$ & $\begin{array}{c}\text { Final } \\
\text { Viscosity } \\
\text { (BU) }\end{array}$ & $\begin{array}{c}\text { Breakdown } \\
\text { (BU) }\end{array}$ & $\begin{array}{c}\text { Setback } \\
\text { (BU) }\end{array}$ \\
\hline Sample A & $11.3 \mathrm{c}$ & $67 b$ & $230 a$ & $441 b$ & $77 a$ & $286 b$ \\
\hline Sample B & 11.1b & $64 a$ & $261 b$ & $356 a$ & $116 c$ & $212 a$ \\
\hline Sample C & $10.9 a$ & $71 \mathrm{c}$ & $276 c$ & $537 \mathrm{c}$ & $75 a$ & $365 \mathrm{c}$ \\
\hline
\end{tabular}

2 Means followed by different letters in a column are significantly different at $\mathrm{p}<0.05$. 
1 Table 3. Cooking and textural properties of Pizzoccheri samples. 


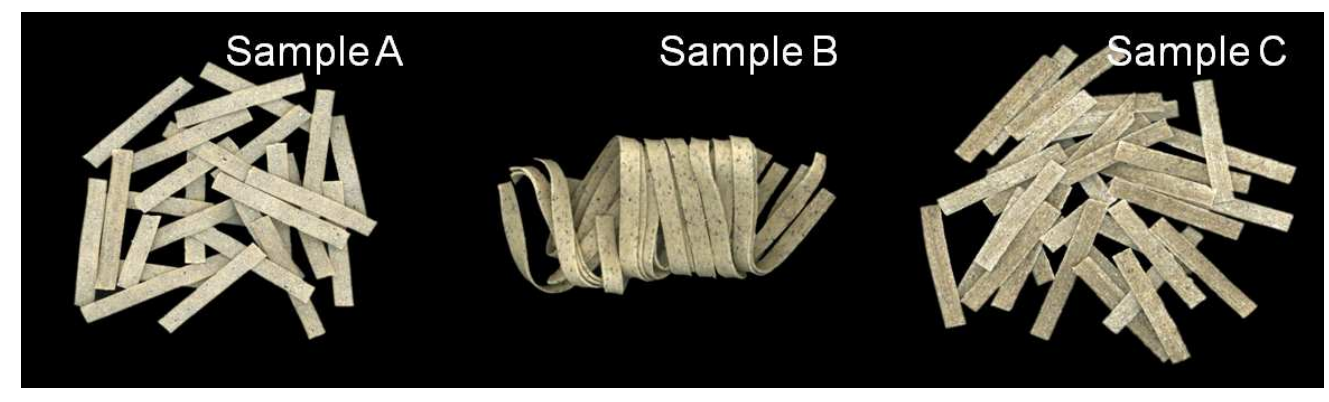

Figure 1. Pizzoccheri shape. $196 \times 56 \mathrm{~mm}(150 \times 150 \mathrm{DPI})$ 


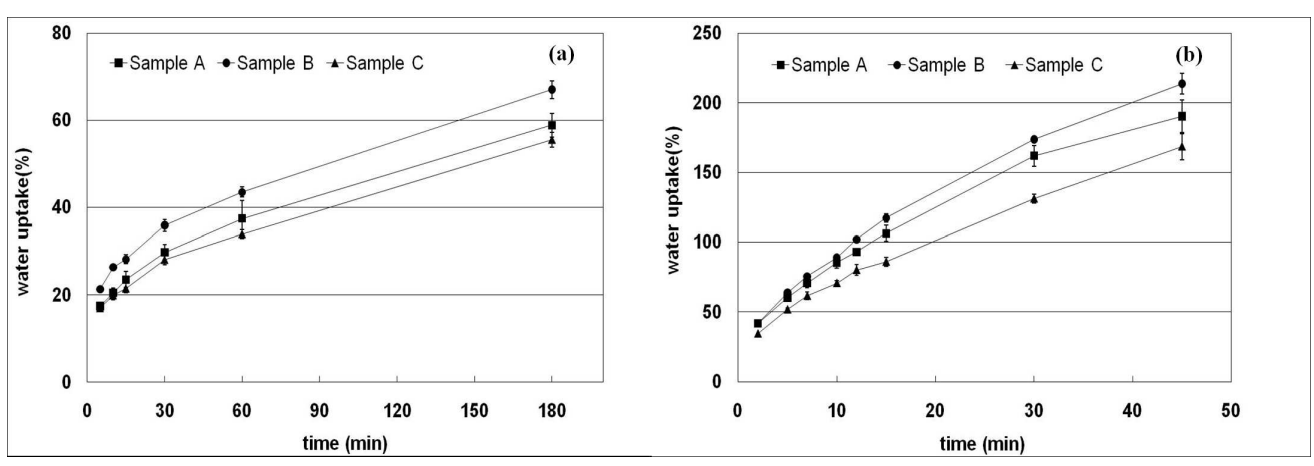

Figure 2. Absorption kinetics at $25^{\circ} \mathrm{C}$ (a) and $90^{\circ} \mathrm{C}$ (b) of Pizzoccheri samples. $368 \times 124 \mathrm{~mm}(150 \times 150 \mathrm{DPI})$ 
Figure 3. Sample images: colour images of Pizzoccheri before (a) and after (d) cooking; 2D and 3D representation of grey level surface distribution before $(b ; c)$ and after cooking $(e ; f)$. $317 \times 169 \mathrm{~mm}(150 \times 150 \mathrm{DPI})$ 


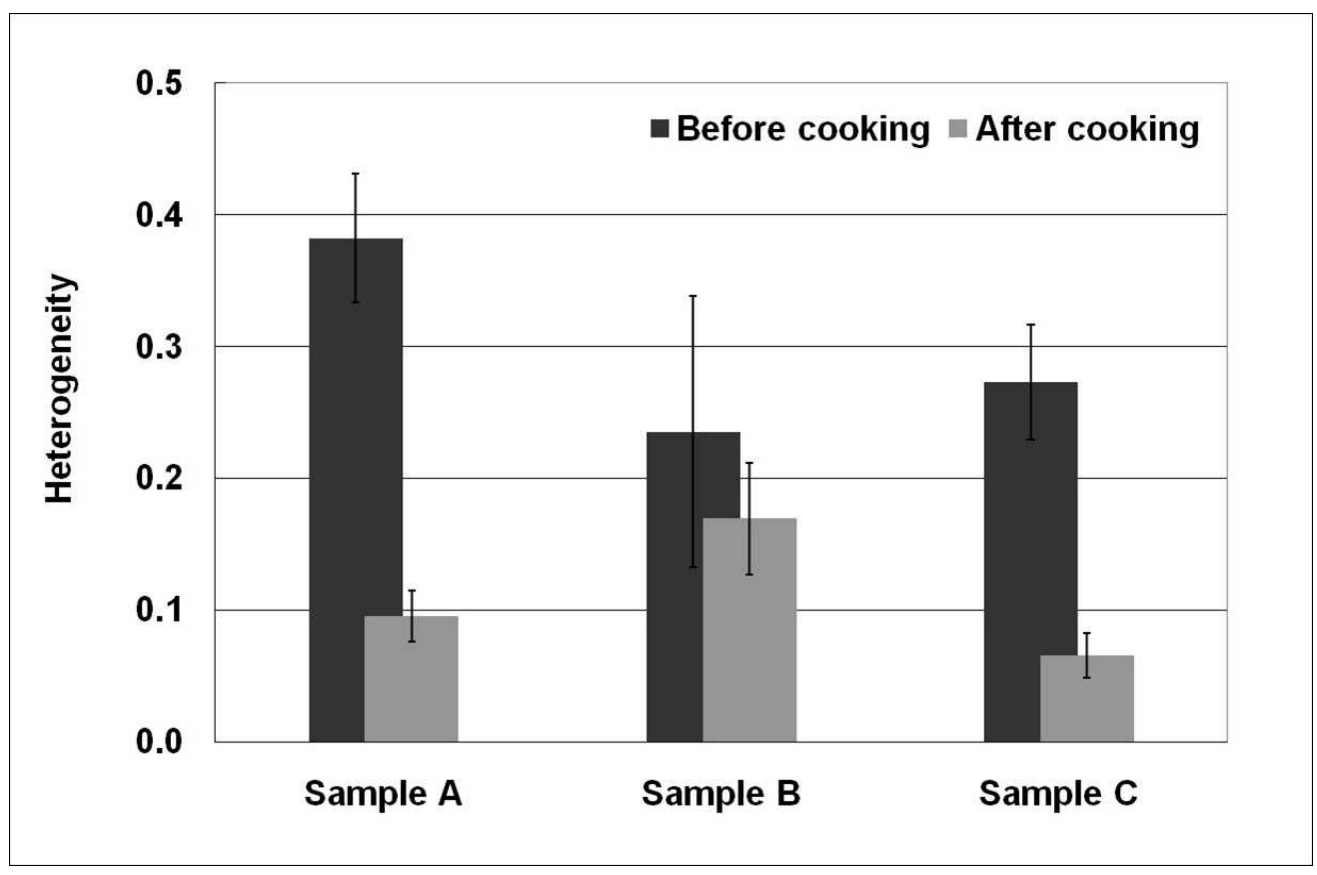

Figure 4. Heterogeneity of Pizzoccheri before and after cooking. $259 \times 169 \mathrm{~mm}(120 \times 120 \mathrm{DPI})$ 